\title{
Multimodal approach of electrotherapy versus myofascial release in patients with chronic mechanical neck pain: a randomized controlled trial
}

\author{
DOI: https://doi.org/10.5114/pq.2019.87735
}

\author{
Mohammed H. El-Gendy', Yasser R. Lasheen ${ }^{1}$, Wafaa K.S. Rezkalla ${ }^{2}$ \\ ${ }^{1}$ Department of Basic Science, Faculty of Physical Therapy, Cairo University, Cairo, Egypt \\ ${ }^{2}$ Department of Physical Therapy, Shoubra General Hospital, Ministry of Health and Population, Cairo, Egypt
}

\section{Abstract}

Introduction. To investigate and compare the effect of the multimodal approach of electrotherapy and myofascial release on pain, range of motion, and functional restriction in patients with chronic mechanical neck pain.

Methods. Overall, 60 patients of both genders, aged 18-40 years, with chronic mechanical neck pain were diagnosed by an orthopaedist in Shoubra General Hospital, Cairo, Egypt. They were randomly assigned into 3 groups. In group A, 20 patients received multimodal approach of electrotherapy (low level laser therapy, interferential therapy, ultrasound, and non-guideline approach in the form of stretch and strength) 3 times a week for 4 weeks. In group B, 20 patients received myofascial release therapy and non-guideline approach in the form of stretch and strength 3 times a week for 4 weeks. In group C, 20 patients received traditional therapeutic exercises in the form of stretch and strength 3 times a week for 4 weeks. All patients in all groups were evaluated pre- and post-treatment through visual analogue scale, cervical range of motion (CROM) device, and neck disability index to assess pain, CROM, and functional restriction.

Results. Multiple pairwise comparison tests (post-hoc tests) revealed that there was no significant difference between the effect of multimodal approach of electrotherapy (group A) and myofascial release therapy (group B) on pain intensity level, CROM, or functional restriction. However, these turned out significantly more effective than traditional therapeutic exercises (group C).

Conclusions. Both multimodal approach of electrotherapy and myofascial release therapy are effective in treating patients with chronic mechanical neck pain.

Key words: multimodal approach, myofascial release, mechanical neck pain

\section{Introduction}

Neck pain is a common musculoskeletal disorder in the modern society; $67 \%$ of the population suffer from it at least once in a lifetime and the prevalence is about $23 \%$. The pain exacerbates and fades periodically, and many patients do not fully recover from the symptoms. Neck pain can be due to musculoskeletal trauma, systemic or inflammatory diseases, neoplasm, or poor posture [1, 2].

Most cases of pain in the neck region are of mechanical origin. The annual incidence of episodes of mechanical neck pain is estimated at 12 per 1000 subjects with a primary care medical appointment, making it one of the main health problems seen by physiotherapy units in primary care [3].

Patients with mechanical neck pain present with localized and/or referred pain, commonly accompanied by point tenderness and restricted cervical range of motion (CROM). The decreased movement of the upper cervical spine can cause excessive movement of the lower cervical spine, increase fatigue in the sternocleidomastoid, anterior scalenus, and upper trapezius, lead to changes of neck postures and breathing patterns, and decrease the range of motion (ROM). Patients with chronic neck pain experience functional impairments including weakening of deep bending neck muscles due to the activation of neck surface muscles, increased deformity of the forward head posture, proprioception impairment, and poor balance. In addition, reduced movement of the cervical spine restricts the ROM of the spine and decreases breathing function $[4,5]$.

Chronic neck pain treatment includes drugs, such as nonsteroidal anti-inflammatory and pain-modulating therapies, which have significant side effects. Nonpharmacological interventions may include cervical massage, acupuncture, neck stretching and training, manual therapy, and low level laser therapy (LLLT). Physiotherapists use methods such as electrotherapy, therapeutic exercise, and manual therapy to intervene in neck pain $[6,7]$.

Multimodal treatment is a combination of at least 2 different therapy modalities, for example exercises combined with mobilisation and medication. Studies concluded that combined physiotherapy treatment involving active and passive modalities was effective for improvement in pain intensity, disability, and quality of life in patients with mechanical neck pain $[8,9]$.

Myofascial release refers to a manual massage technique that is performed for stretching the fascia and releasing bonds between fascia and skin, muscles and bones, with the aim of relieving pain, increasing ROM and body balance. It is said that the effect of this technique can be the mechanical, neural facilitation and psycho-physiological adaptation [10,11]. Also, the need for this study arises from lack of high-powered trials with appropriate outcome measures examining the effect of the multimodal approach of electrotherapy and myofascial release on pain, $\mathrm{ROM}$ and functional restriction in

Correspondence address: Wafaa K.S. Rezkalla, Department of Physical Therapy, Shoubra General Hospital, Ministry of Health and Population, 7 Ahmed Elziat Street, Bean Elsariat, El Dokki, Giza, Egypt, e-mail: wafawafaakamel88@gmail.com 
patients with chronic mechanical neck pain. Therefore, the study was conducted to investigate if there was a difference between the efficacy of multimodal approach of electrotherapy and myofascial release in treatment of patients with chronic mechanical neck pain.

\section{Subjects and methods}

\section{Design}

The study was designed as a prospective, randomized, controlled trial. It was conducted between October 2017 and May 2018.

\section{Participants}

A sample of 60 patients of both genders were recruited from the Orthopedic Outpatient Clinic, Shoubra General Hospital, Cairo, Egypt. The inclusion criteria comprised mechanical neck pain for at least 3 months with or without shoulder girdle and upper limb unilateral or bilateral symptoms and myofascial trigger points. The participants' age ranged from 18 to 40 years, their body mass index from 18.5 to $24.9 \mathrm{~kg} / \mathrm{m}^{2}$. Subjects were excluded if there was a positive neurological examination result (presence of positive motor, reflex, or sensory abnormalities indicating spinal root compression) or abnormal neurological signs in the upper limbs relating to nerve entrapment, inflammation, infection, or advanced degeneration due to a systemic rheumatologic disease (e.g., rheumatoid arthritis), congenital malformation, trauma, cerebrovascular abnormalities, cervical spine surgery or stenosis, metabolic or systemic disorders, cancer, known photosensitivity or other illnesses unrelated to neck pain which precluded involvement for practical reasons, pregnancy.

\section{Randomization}

Each participant was informed on the nature, purpose, and benefits of the study, on their right to refuse or withdraw at any time, and on the confidentiality of any obtained data. The patients were randomly assigned into 3 equal groups (control group and 2 study groups) with the use of a computer-based randomization program. No subject dropped out from the study after randomization. The patients were blinded about which group they were allocated by an independent researcher.

\section{Interventions}

The participants were randomly assigned into 3 groups. Group A, composed of 20 patients, received multimodal approach of electrotherapy (LLLT, interferential therapy [IFT], ultrasound [US], and non-guideline approach in the form of stretch and strength) for 4 weeks. Group B, composed of 20 patients, received myofascial release therapy and traditional therapeutic exercises in the form of stretch and strength for 4 weeks. Group C, composed of 20 patients, received nonguideline approach in the form of stretch and strength for 4 weeks.

\section{Ultrasound therapy}

Group A received continuous US waves of $1 \mathrm{MHz}$ frequency and $1-1.5 \mathrm{~W} / \mathrm{cm}^{2}$ power as for chronic cases. US was performed bilaterally to cover the trapezius muscle over 8 minutes. The dosage was adjusted to the anatomical re- gion of the neck. The treatment was applied by using circular movements with a $5-\mathrm{cm}^{2}$ US head, 3 sessions per week for 4 weeks.

\section{Low level laser therapy}

Group A received LLLT by low-level diode laser emitting a divergent $905-\mathrm{nm}$ (red) laser light generating a $25-\mathrm{mW}$ output. LLLT was used at trigger points at bilateral shoulders during passive external rotation of the shoulder encompassing the anterior muscles of the shoulder, the pectoralis group, bilateral cervical muscles, trapezius muscles during passive lateral flexion of the cervical spine with the subject's head in the neutral position, bilateral sternocleidomastoid and scalene muscles during passive ROM. Each site was treated for 1 minute, which provided a total treatment time of 12 minutes with $1.5 \mathrm{~J} / \mathrm{cm}^{2}$ power, 3 sessions per week for 4 weeks.

\section{Interferential therapy}

Group A received interferential current modality with a Zimmer machine for IFT application. Four electrodes were placed around the upper trapezius muscle. A pair of electrodes with a current frequency of $4000 \mathrm{~Hz}$ crossed another pair of electrodes with a current frequency of $4100 \mathrm{~Hz}$ to stimulate the target muscle in a criss-cross current pattern from prone position. Parameters used were $0-100 \mathrm{~Hz}$ beat frequency, intensity $(\mathrm{mA})$ set at the tactile sensation threshold, $20 \mathrm{~min}$ utes on each occasion, 3 sessions per week for 4 weeks.

\section{Myofascial release therapy}

Group B received myofascial release applied from sitting position after exact determination of the pain location; for obtaining general relaxation, superficial stroke massage was performed for 2-3 minutes on the back region to the neck and shoulders area in reciprocating and transverse way. Then the therapist focused on the pain region locally and applied myofascial release technique with pressure proper with the patient's pain tolerance. At the end of the treatment session, about 2-3-minute surface stroke massage was performed again and the treatment was ended. Each treatment session took 20 minutes; there were 3 sessions per week for 4 weeks.

\section{Non-guideline approach}

All the patients in the 3 groups received non-guideline approach in the form of stretch and strength, 3 sessions per week for 4 weeks, as follows:

- Gentle stretching of the pectoral muscle. The patient was sitting with the hands behind the head. The therapist stood behind the patient and grasped their elbows, had the patient breathe in as they brought the elbows out to the side (horizontal abduction and scapular adduction). The therapist held the elbows at this end-point as the patient breathed out. No forceful stretch was needed against the elbows because the rib cage elongated the proximal attachment of the pectoralis major muscles bilaterally. As the patient repeated the inhalation, the therapist again moved the elbows up and out to the end of the available range and held as the patient breathed out. Three times for 30 seconds depending on the patient's tolerance.

- Gentle stretching of the trapezius muscle. The patient was sitting with the head rotated to the tight side. The therapist stood behind the patient and applied the stretch by adding 
a combination of cervical flexion, further rotation to the tight side, and side bending away from the tight side. A more aggressive manual stretch could be performed by using the other hand to depress the distal clavicle and the scapula. Three times for 30 seconds depending on the patient's tolerance.

- Gentle stretching of the scaleni muscles. The patient was sitting. The patient first performed axial extension (tucked the chin and straightened the neck) and then side-bended the neck opposite and rotated it toward the tight muscles. The therapist stood behind the patient and stabilized the head with the one hand around the side of the patient's head and face, held the head against their trunk or shoulder, and placed the other hand across the top of the rib cage on the side of tightness. He instructed the patient to inhale and exhale, applying a downward pressure (resisting elevation of the rib cage) as the patient inhaled again. As the patient relaxed (exhaled), the therapist took up the slack. Three times for 30 seconds depending on the patient's tolerance.

- Gentle stretching of the levator scapulae muscle. The patient was sitting with the head rotated opposite to the side of tightness (looking away from the tight side) and forward bent until a slight pull was felt in the posterolateral aspect of the neck. The arm on the side of tightness was abducted, and the hand was placed behind the head to help stabilize it in the rotated position. The therapist stood behind the patient and stabilized with one arm, placed the other hand (same side as the tight muscle) over the superior angle of the scapula. With the muscle now in its stretched position, he told the patient to breathe in, then out. He held the shoulder and scapula down to maintain the stretch as the patient breathed in again (they contracted the muscle against the resistance of the fixating hand). To increase the stretch, the therapist pressed down against the superior angle of the scapula. Three times for 30 seconds depending on the patient's tolerance.

- Gentle stretching of the suboccipital muscle. The patient was sitting. The therapist identified the spinous process of the second cervical vertebra and stabilized it with his thumb or with the second metacarpophalangeal joint (and the thumb and index finger around the transverse processes). He told the patient to slowly nod, doing just a tipping motion of the head on the upper spine. He guided the movement by placing the other hand across the patient's forehead. Three times for 30 seconds depending on the patient's tolerance.

- Strengthening exercises consisted of cervical flexion and extension. For flexion strength: the therapist had the patient sit, kept his hands on the forehead, and pressed the forehead into the palms in a nodding fashion while not moving, holding for 3 seconds; 3 sets of 10 repetitions with a 60 -second rest period between sets. For extension: the therapist had the patient press the back of the head into hand, placed in the back, near the top of the head.

- Shoulder retraction exercise. The patient was sitting with shoulders abducted at $90^{\circ}$, elbows flexed, and forearms parallel to the floor. The therapist instructed the patient to perform horizontal head and shoulder retraction: abduction with scapular retraction and hold for 3 seconds. Three sets of 10 repetitions with a 60 -second rest period between sets.

- Seated upright rowing with resistance tubing. The patient was sitting and grasping with resistance tubing, and pulled backward with the arms in a rowing action and hold for 3 seconds; 3 sets of 10 repetitions with a 60 -second rest period between sets.

- Push-ups if tolerated. The patient was sitting with both hands on blocks. The therapist asked the patient to push down on the hands and lift the body. After the elbows were fully extended, he emphasized scapular depression and held for 3 seconds. Three sets of 10 repetitions with a 60 -second rest period between sets.

\section{Outcome measures}

\section{Visual analogue scale}

Visual analogue scale (VAS) is a line used to describe the subjective pain level. It is marked from 0 to 10 , with 0 representing no pain and 10 standing for unbearable pain. The subjects were instructed to mark the level of pain that they were experiencing. It was performed for all the patients in all groups before and after the treatment.

\section{Cervical range of motion}

It was assessed for all the patients in all groups before participation in the study and after the end of the treatment.

The device is made of an unbreakable polyester material; it has a comfortable frame that adjusts to the patient's head and is secured with Velcro straps, and there are 3 plane meters preset to the frame. The sagittal plane meter measures flexion and extension, while the lateral plane meter measures lateral flexion; both are fluid damped inclinometers and work by the force of gravity as the position of the head changes. The magnetic plane meter measures rotational movement in conjugation with magnetic yoke.

The CROM device was mounted over the patient's nose bridge and ears, then secured to the head with a Velcro strap. Each patient was instructed to move their head to the end point of their active ROM for each of 6 cervical spine movements. Flexion and extension occurred in the sagittal plane, right and left lateral flexions occurred in the frontal plane, and right and left rotations occurred in the transverse plane. Cervical flexion and extension were measured by the inclinometer placed above the ear. During flexion, each patient was verbally instructed to bend their head forward as far as possible until feeling tightness or pain but without bringing their shoulders away from the chair's backrest; for extension, patients were verbally instructed to lift the chin and bend their head back as far as possible until feeling tightness or pain but without putting extra pressure against the backrest with their shoulders. Left and right lateral bending were measured by an inclinometer placed on the forehead above the bridge of the nose while the patient tilted their head as far as possible until feeling tightness or pain but without lifting the other shoulder, and cervical rotation was recorded with the magnetic plane meter in unison with the shoulder-mounted magnetic yoke. Each patient was instructed to keep their shoulders against the chair backrest and to turn only their head.

\section{Neck disability index}

The neck disability index (NDI) was assessed for all patients before and after the treatment. NDI was developed to measure self-perceived disability from neck pain. It is a 10item scaled questionnaire, and the patients were asked to make a mark in each section which most closely described their problem. If a patient identified with 2 or more statements in any one section, they were asked to only mark the box which most closely described their problems. Each item was recorded out of 5 for a maximum total score of 50 . The questionnaire was performed before and after treatment in all groups. 


\section{Statistical analysis}

Results are expressed as mean \pm standard deviation or median (minimum-maximum). The mean median difference was calculated as follows:

$$
\text { difference }=\text { pre-treatment }- \text { post-treatment }
$$

Comparisons between mean values of variables in the 3 groups (control group and 2 study groups) were performed by using multiple pairwise; also, pairwise comparison (pretreatment vs. post-treatment) within the same group was performed with post-hoc tests. Comparisons between median values of the 3 groups (control group and 2 study groups) were performed by using post-hoc tests; also, pairwise comparison (pre-treatment vs. post-treatment) within the same group was carried out. The Statistical Package for the Social Sciences (SPSS) computer program (version 23 for Windows) was used for data analysis. The value of $p \leq$ 0.05 was considered significant and $p<0.01$ was considered highly significant.

\section{Ethical approval}

The research related to human use has complied with all the relevant national regulations and institutional policies, has followed the tenets of the Declaration of Helsinki and the Consolidated Standards of Reporting Trials, and has been approved by the institutional review board at Faculty of Physical Therapy, Cairo University.

\section{Informed consent}

Informed consent has been obtained from all individuals included in this study.

\section{Results}

A total of 60 patients were randomized for study intervention. Group A consisted of 20 patients and received multimodal approach of electrotherapy (LLLT, IFT, US, and traditional therapeutic exercises in the form of stretch and strength) for 4 weeks. Group B consisted of 20 patients and received myofascial release therapy and traditional therapeutic exercises in the form of stretch and strength for 4 weeks. Group $\mathrm{C}$ consisted of 20 patients and received traditional therapeutic exercises in the form of stretch and strength for 4 weeks. All randomized patients completed the trial, as shown in Figure 1. The groups were similar at baseline $(p>0.05)$ with regard to age (Table 1$)$.

Statistical analysis using $3 \times 2$ mixed design analysis of variance (MANOVA) indicated that there were significant effects of the tested group (the first independent variable) on all the tested dependent variables: VAS, NDI, ROM of neck flexion, extension, right and left side bending and rotation ( $F=4.03, p=0.0001)$. In addition, there were significant effects of the measuring periods (the second independent variable) on the tested dependent variables ( $F=126.91, p=$ 0.0001). The interaction between the 2 independent variables was significant, which indicates that the effect of the tested group (first independent variable) on the dependent variables was influenced by the measuring periods (second independent variable) $(F=8.149, p=0.0001)$. In the same context regarding within-group effects, the multiple pairwise comparison tests revealed that there was a significant reduction $(p>$ $0.05)$ in the VAS and NDI scores and an increase $(p>0.05)$ in neck flexion and extension, right and left side bending, right and left side rotation post-treatment compared with pre-treat-

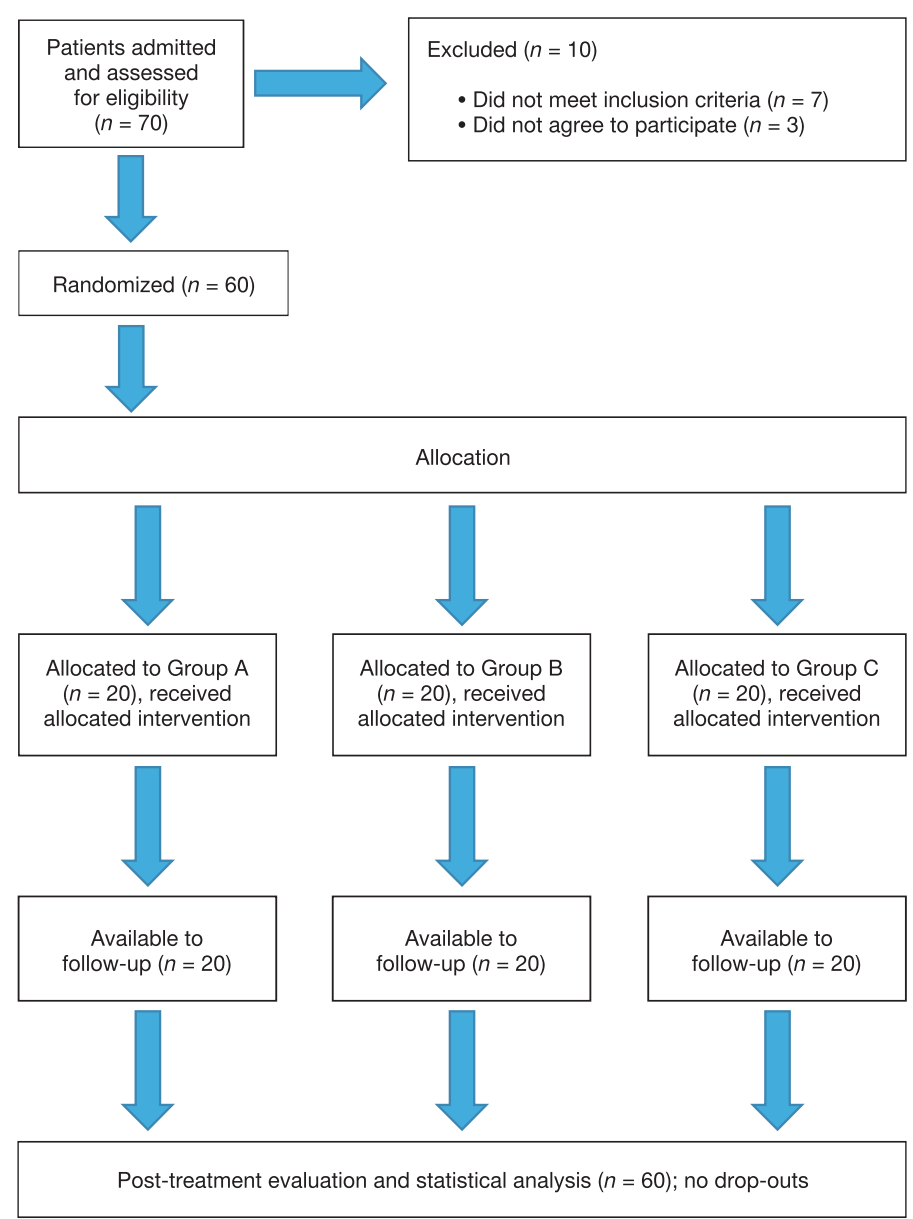

Figure 1. Flow chart of the randomized trial in accordance with the Consolidated Standards of Reporting Trials

Table 1. Descriptive statistics and one-way analysis of variance for the mean age values in the 3 tested groups

\begin{tabular}{|c|c|c|c|c|c|}
\cline { 2 - 6 } \multicolumn{1}{c|}{} & $\begin{array}{c}\text { Group A } \\
(n=20)\end{array}$ & $\begin{array}{c}\text { Group B } \\
(n=20)\end{array}$ & $\begin{array}{c}\text { Group C } \\
(n=20)\end{array}$ & F-value & $p$-value \\
\hline $\begin{array}{c}\text { Age } \\
\text { (years) }\end{array}$ & $34.8 \pm 5.67$ & $33.9 \pm 5.51$ & $33.65 \pm 5.7$ & 0.231 & 0.795 \\
\hline
\end{tabular}

ment in the groups. As for the between-group multiple pairwise comparisons, the tests implied that there was no significant difference between groups $\mathrm{A}$ and $\mathrm{B}(p>0.5)$. There was, however, a significant difference between group A vs. group $C$ and between group $B$ vs. group $C(p<0.05)$, with a significant reduction in favour of groups $A$ and $B$ in all dependent variables (Tables 2 and 3 ).

\section{Discussion}

In terms of number of years lived with disability, back and neck pain is the most important condition worldwide and usually runs an episodic course over a person's lifetime. Neck pain is a significant individual, social, and economic health problem, affecting up to $2 / 3$ of adults at some point in their lives [12].

Neck pain, after low back pain, is one of the most common pain disorders of the musculoskeletal system. In chronic cases, symptoms persist, causing severe discomfort, inability to work, and a decreased quality of life [13].

In the available literature, there have been no studies in this area, and the gap in research might be filled by this study, adding information for physical therapists in governmental 
Table 2. Descriptive statistics for all dependent variables for all groups at different measuring points

\begin{tabular}{|l|c|r|r|r|r|r|}
\hline \multirow{2}{*}{ Variable } & \multicolumn{2}{|c|}{ Group A } & \multicolumn{2}{c|}{ Group B } & \multicolumn{2}{c|}{ Group C } \\
\cline { 2 - 7 } & \multicolumn{1}{|c|}{ Pre } & \multicolumn{1}{c|}{ Post } & Pre & Post & Pre & Post \\
\hline Visual analogue scale & $6.8 \pm 0.76$ & $3.65 \pm 0.98$ & $6.65 \pm 0.87$ & $3.4 \pm 1.87$ & $6.5 \pm 0.82$ & $4.95 \pm 0.99$ \\
\hline Neck flexion (degrees) & $44.45 \pm 3.8$ & $57.4 \pm 4.86$ & $44.65 \pm 3.71$ & $57.4 \pm 3.74$ & $44.9 \pm 3.68$ & $48.55 \pm 4.14$ \\
\hline Neck extension (degrees) & $54.4 \pm 4.32$ & $66.25 \pm 4.19$ & $54.55 \pm 4.22$ & $67.65 \pm 5.47$ & $54.5 \pm 4.19$ & $57.05 \pm 4.26$ \\
\hline Neck right side bending (degrees) & $36.25 \pm 4.17$ & $47.9 \pm 5.7$ & $36.9 \pm 4.15$ & $47.05 \pm 5.44$ & $36.7 \pm 4.14$ & $41.5 \pm 4.09$ \\
\hline Neck left side bending (degrees) & $37.05 \pm 3.73$ & $46.05 \pm 5.99$ & $36.85 \pm 3.92$ & $46.9 \pm 6.91$ & $36.75 \pm 3.9$ & $41.4 \pm 3.18$ \\
\hline Neck right rotation (degrees) & $55.25 \pm 4.72$ & $71.25 \pm 6.25$ & $55.75 \pm 4.66$ & $69.75 \pm 6.58$ & $54.75 \pm 4.72$ & $60 \pm 6.68$ \\
\hline Neck left rotation (degrees) & $55.5 \pm 4.55$ & $67 \pm 8.17$ & $55 \pm 4.86$ & $65.5 \pm 6.26$ & $55.25 \pm 4.72$ & $58.25 \pm 5.44$ \\
\hline Neck disability index & $24.8 \pm 3.84$ & $15.5 \pm 6.4$ & $24.85 \pm 3.82$ & $15.35 \pm 5.87$ & $24.7 \pm 3.78$ & $21.8 \pm 4.03$ \\
\hline
\end{tabular}

Values of all dependent variables are expressed as mean $\pm S D$.

Table 3. Comparison tests $p$-values for all dependent variables in groups

\begin{tabular}{|c|c|c|c|c|c|c|c|c|}
\hline \multicolumn{9}{|l|}{ Within groups } \\
\hline Group & $\begin{array}{l}\text { Visual ana- } \\
\text { logue scale }\end{array}$ & $\begin{array}{l}\text { Neck } \\
\text { flexion }\end{array}$ & $\begin{array}{c}\text { Neck } \\
\text { extension }\end{array}$ & $\begin{array}{l}\text { Neck right } \\
\text { side bending }\end{array}$ & $\begin{array}{c}\text { Neck left } \\
\text { side bending }\end{array}$ & $\begin{array}{l}\text { Neck right } \\
\text { rotation }\end{array}$ & $\begin{array}{c}\text { Neck left } \\
\text { rotation }\end{array}$ & $\begin{array}{l}\text { Neck dis- } \\
\text { ability index }\end{array}$ \\
\hline A & $0.001^{*}$ & $0.001^{*}$ & $0.001^{*}$ & $0.001^{*}$ & $0.001^{*}$ & $0.001^{*}$ & $0.001^{*}$ & $0.001^{*}$ \\
\hline B & $0.001^{*}$ & $0.001^{*}$ & $0.001^{*}$ & $0.001^{*}$ & $0.001^{*}$ & $0.001^{*}$ & $0.001^{*}$ & $0.001^{*}$ \\
\hline C & $0.001^{*}$ & $0.001^{*}$ & 0.058 & $0.001^{*}$ & $0.001^{*}$ & $0.002^{*}$ & 0.084 & $0.015^{\star}$ \\
\hline \multicolumn{9}{|l|}{ Between groups } \\
\hline Variable & \multicolumn{2}{|c|}{ Measurement } & \multicolumn{2}{|c|}{ Group A vs. group B } & \multicolumn{2}{|c|}{ Group A vs. group C } & \multicolumn{2}{|c|}{ Group B vs. group C } \\
\hline \multirow{2}{*}{ Visual analogue scale } & \multicolumn{2}{|c|}{ Pre } & \multicolumn{2}{|c|}{0.567} & \multicolumn{2}{|c|}{0.255} & \multicolumn{2}{|c|}{0.567} \\
\hline & \multicolumn{2}{|c|}{ Post } & \multicolumn{2}{|c|}{0.46} & \multicolumn{2}{|c|}{$0.001^{*}$} & \multicolumn{2}{|c|}{$0.001^{*}$} \\
\hline \multirow{2}{*}{ Neck flexion } & \multicolumn{2}{|c|}{ Pre } & \multicolumn{2}{|c|}{0.867} & \multicolumn{2}{|c|}{0.706} & \multicolumn{2}{|c|}{0.834} \\
\hline & \multicolumn{2}{|c|}{ Post } & \multicolumn{2}{|c|}{0.999} & \multicolumn{2}{|c|}{$0.001^{*}$} & \multicolumn{2}{|c|}{$0.001^{*}$} \\
\hline \multirow{2}{*}{ Neck extension } & \multicolumn{2}{|c|}{ Pre } & \multicolumn{2}{|c|}{0.911} & \multicolumn{2}{|c|}{0.941} & \multicolumn{2}{|c|}{0.97} \\
\hline & $\mathrm{Po}$ & & & 348 & 0.0 & & & $001^{*}$ \\
\hline & $\mathrm{Pr}$ & & & 623 & 0.7 & & & 88 \\
\hline 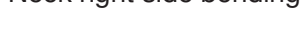 & Po & & & 602 & 0.0 & & & $001^{*}$ \\
\hline & $\mathrm{Pr}$ & & & 87 & 0.8 & & & 935 \\
\hline & Po & & & 633 & 0.0 & & & $003^{*}$ \\
\hline & $\mathrm{Pr}$ & & & 738 & 0.7 & & & 504 \\
\hline 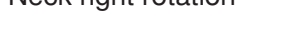 & $\mathrm{Po}$ & & & 469 & 0.0 & & & $001^{*}$ \\
\hline & $\mathrm{Pr}$ & & & 739 & 0.8 & & & 868 \\
\hline & Po & & & 484 & 0.0 & & & $001^{*}$ \\
\hline & $\mathrm{Pr}$ & & & 967 & 0.9 & & & 902 \\
\hline & $\mathrm{Po}$ & & & 932 & 0.0 & & & $001^{*}$ \\
\hline
\end{tabular}

* Significant at the level of alpha $<0.05$

hospitals and private clinics about which is more effective: the multimodal approach of electrotherapy or myofascial release on pain, ROM of cervical spine and functional restriction in treatment of chronic mechanical neck pain when combined with traditional therapeutic exercises.

The study showed that multimodal approach of electrotherapy and traditional therapeutic exercises in the form of stretch and strength on the one hand and myofascial release stretch and strength on the other had a significant effect on improving pain intensity level, CROM, and neck functional restriction.

The obtained results remain in line with those of a study by Hou et al. [14], who investigated the effect of many forms of combined therapy and concluded that therapeutic combinations such as hot pack plus active ROM and stretch with spray; hot pack plus active ROM and stretch with spray, as well as transcutaneous electric nerve stimulation; and hot 
pack plus active ROM and IFT, as well as myofascial release technique are most effective for easing myofascial trigger point pain and increasing CROM.

Mukkannavar [15] compared between combination therapy (transcutaneous electric nerve stimulation and US) and ischaemic compression in the treatment of active myofascial trigger points and showed that combination therapy resolved acute active trigger points pain and increased ROM more rapidly than the ischaemic compression treatment technique.

Also, the results of this study agree with those obtained by Namvar et al. [16], who concluded that myofascial release was one of the effective manual therapy techniques in reducing pain and disability, as well as improving the isometric extension strength of neck in patients with non-specific chronic neck pain. Hamoda et al. [17] reported myofascial release as an effective method for improving electrophysiological and clinical measures among pregnant women with carpal tunnel syndrome.

Moreover, Ravish et al. [18] compared the effectiveness of myofascial release technique vs. positional release technique with laser in patients with unilateral trapezitis. A total of 60 subjects with unilateral upper trapezius spasm were randomly allocated into 2 groups: $A$ and $B$. Laser treatment was common to both groups; myofascial release technique was applied in group A and positional release therapy in group B for alternatively 3 days for 4 weeks. The authors concluded that both groups showed a significant improvement in pain reduction, functional limitation, and ROM. However, myofascial release therapy with laser provided a better improvement than positional release technique with laser when subjects in both groups were compared.

The results of the current study disagreed with RodríguezHuguet et al. [19], who concluded that both myofascial release and physical therapy multimodal programs provided improvement of pain and pressure pain thresholds in patients with neck pain. However, myofascial release could be better than a multimodal program that includes US, transcutaneous electric nerve stimulation, and massage. The effect size was larger for VAS and left and right suboccipital points, and medium for left and right upper trapezius points. Overall, the improvement of pressure pain thresholds provided by myofascial release was $20 \%$ greater than that obtained with a multimodal program, perhaps because the program did not involve LLLT and used transcutaneous electric nerve stimulation instead of IFT.

Roche et al. [6] implied that LLLT was safe and effective for temporary pain relief and improving ROM in patients with chronic pain in the neck and shoulder areas due to osteoarthritis, muscle spasms, and cervical and thoracic spine strain. Combined with chiropractic medicine and physical therapy, LLLT may help patients lead a normal, active, and healthy life without the need for analgesic medications.

Chow and Barnsley [20] performed a meta-analysis to investigate the efficacy of LLLT in the treatment of neck pain. LLLT with infrared wavelengths turned out efficacious for the treatment of neck pain, with limited evidence provided from the reviewed trials. The reduction in pain levels was modest in patients with chronic neck pain, and the outcomes limited by short-term follow-up were supported by positive functional changes.

In their meta-analysis, Gross et al. [21] evaluated LLLT for adults with neck pain and supported the implementation of the technique for chronic neck pain.

Also, Acedo et al. [22] compared muscle relaxation of the upper trapezius induced by the application of transcutaneous electric nerve stimulation and IFT in females with chronic nonspecific neck discomfort to reveal if a decrease in muscular activity could be observed at sensorial level stimulation in medium-frequency current, using current intensity $(\mathrm{mA})$ only in the sensory threshold. The authors found that IFT induced upper trapezius relaxation after 3 sessions in females with neck discomfort, but applying transcutaneous electric nerve stimulation did not change the muscular tension. However, these results should be interpreted carefully owing to the lack of differences between groups. A significant pain decrease was found in the subjects of both groups but only IFT application presented a clinically important improvement.

Cheing and Hui-Chan [23] examined whether transcutaneous electrical nerve stimulation or IFT was more effective in reducing experimentally induced heat pain in young healthy subjects. They concluded that both transcutaneous electrical nerve stimulation and IFT increased the heat pain threshold to a similar extent during stimulation. However, the post-stimulation effect of interferential current lasted longer than that of transcutaneous electrical nerve stimulation.

In summary, the study demonstrated that a comparison between multimodal approach of electrotherapy and myofascial release for their effect on pain, CROM, and functional restriction in the treatment of patients with chronic mechanical neck pain revealed no significant differences between them.

\section{Limitations}

Although the current study reveals objective data with statistically significant differences, there are some limitations. The main one is the short study duration. Therefore, longitudinal studies are needed to evaluate long-term effects of multimodal approach of electrotherapy or myofascial release on pain, CROM, and functional restriction in treatment of chronic mechanical neck pain.

\section{Conclusions}

The multimodal approach of electrotherapy and traditional therapeutic exercises in the form of stretch and strength and, on the other hand, myofascial release therapy and traditional therapeutic exercises in the form of stretch and strength are both effective with no significant difference in treating patients with chronic mechanical neck pain. This improvement was reflected in the significant decrease in VAS assessment for pain, increase of CROM, and drop in NDI as a measure of functional restriction in group $A$ and group $B$.

\section{Acknowledgments}

We would like to thank all individuals who contributed to the completion of this work, especially the study participants.

\section{Disclosure statement}

No author has any financial interest or received any financial benefit from this research.

\section{Conflict of interest}

The authors state no conflict of interest.

\section{References}

1. Xiaoqiang Z, Shusheng T, Qiangmin H. Understanding of myofascial trigger points. Chin Med J. 2014;127(24): 4271-4277; doi:10.3760/cma.j.issn.0366-6999.20141999.

2. Hoy DG, Protani M, De R, Buchbinder R. The epidemiology of neck pain. Best Pract Res Clin Rheumatol. 2010; 24(6):783-792; doi: 10.1016/j.berh.2011.01.019. 
3. González Rueda V, López de Celis C, Barra López ME, Carrasco Uribarren A, Castillo Tomás S, Hidalgo García C. Effectiveness of a specific manual approach to the suboccipital region in patients with chronic mechanical neck pain and rotation deficit in the upper cervical spine: study protocol for a randomized controlled trial. BMC Musculoskelet Disord. 2017;18(1):384; doi: 10.1186/ s12891-017-1744-5.

4. Lee J-H. Effects of forward head posture on static and dynamic balance control. J Phys Ther Sci. 2016;28(1): 274-277; doi: 10.1589/jpts.28.274.

5. Dimitriadis Z, Kapreli E, Strimpakos N, Oldham J. Respiratory weakness in patients with chronic neck pain. Man Ther. 2013;18(3):248-253; doi: 10.1016/j.math.2012. 10.014.

6. Roche GC, Murphy DJ, Berry TS, Shanks S. Low-level laser therapy for the treatment of chronic neck and shoulder pain. Funct Neurol Rehabil Ergon. 2016;6(2):97-104.

7. Masaracchio M, Cleland JA, Hellman M, Hagins M. Shortterm combined effects of thoracic spine thrust manipulation and cervical spine nonthrust manipulation in individuals with mechanical neck pain: a randomized clinical trial. J Orthop Sports Phys Ther. 2013;43(3):118-127; doi: 10.2519/jospt.2013.4221.

8. Tsakitzidis G, Remmen R, Dankaerts W, Van Royen P. Non-specific neck pain and evidence-based practice. Eur Sci J. 2013;9(3):1-19; doi: 10.19044/esj.2013.v9n $3 p \% 25 p$.

9. Soysal ANO, Aslan UB. Treatment of chronic neck pain by two combined physiotherapy programs: comparison of phonophoresis and ultrasound. Asian Biomed. 2013; 7(6):821-827; doi: 10.5372/1905-7415.0706.245.

10. Bakar Y, Sertel M, Oztürk A, Yümin ET, Tatarli N, Ankarali $\mathrm{H}$. Short term effects of classic massage compared to connective tissue massage on pressure pain threshold and muscle relaxation response in women with chronic neck pain: a preliminary study. J Manipulative Physiol Ther. 2014;37(6):415-421; doi: 10.1016/j.jmpt.2014. 05.004 .

11. Shah S, Bhalara A. Myofascial release. Int $\mathrm{J}$ Health Sci Res. 2012;2(2):69-77.

12. Vos T, Allen C, Arora M, Barber RM, Bhutta ZA, Brown A, et al. Global, regional, and national incidence, prevalence, and years lived with disability for 310 diseases and injuries, 1990-2015: a systematic analysis for the Global Burden of Disease Study 2015. Lancet. 2016;388(10053): 1545-1602; doi: 10.1016/S0140-6736(16)31678-6.

13. Picavet HS, Schouten JS. Musculoskeletal pain in the Netherlands: prevalences, consequences and risk groups, the DMC(3)-study. Pain. 2003;102(1-2):167-178; doi: 10.1016/s0304-3959(02)00372-x.

14. Hou C-R, Tsai L-C, Cheng K-F, Chung K-C, Hong C-Z. Immediate effects of various physical therapeutic modalities on cervical myofascial pain and trigger-point sensitivity. Arch Phys Med Rehabil. 2002;83(10):1406-1414; doi: 10.1053/apmr.2002.34834.

15. Mukkannavar PB. Effect of combination therapy [TENS and ultrasound] and ischemic compression in the treatment of active myofascial trigger points. J Exerc Sci Physiother. 2008;4(2):95-103.

16. Namvar H, Olyaei G, Moghadam BA, Hosseinifar M. Effect of myofascial release technique on pain, disability, maximum isometric contraction of the extensor muscles, and pressure pain threshold in patients with chronic nonspecific neck pain: double blinded randomized clinical
17. Hamoda RE, Osman DA, Hamada HA, Radwan R, Yousef AM, Samea GA, et al. Effect of myofascial release on electrophysiological and clinical measures of pregnant women with carpal tunnel syndrome. Physiother Quart. 2019;27(1):18-24; doi: 10.5114/pq.2019.83057.

18. Ravish VN, Shridhar S, Helen S. To compare the effectiveness of myofascial release technique versus positional release technique with laser in patients with unilateral trapezitis. J Evol Med Dent Sci. 2014;3(9):2161-2166; doi: 10.14260/jemds/2014/2121.

19. Rodríguez-Huguet M, Gil-Salú JL, Rodríguez-Huguet $P$, Cabrera-Afonso JR, Lomas-Vega R. Effects of myofascial release on pressure pain thresholds in patients with neck pain: a single-blind randomized controlled trial. Am J Phys Med Rehabil. 2018;97(1):16-22; doi: 10.1097/ PHM.0000000000000790.

20. Chow RT, Barnsley L. Systematic review of the literature of low-level laser therapy (LLLT) in the management of neck pain. Lasers Surg Med. 2005;37(1):46-52; doi: 10.1 002/lsm.20193.

21. Gross AR, Dziengo S, Boers O, Goldsmith CH, Graham N, Lilge L, et al. Low level laser therapy (LLLT) for neck pain: a systematic review and meta-regression. Open Orthop J. 2013;7:396-419; doi: 10.2174/1874325001307010396.

22. Acedo AA, Luduvice Antunes AC, Barros dos Santos A, Barbosa de Olveira C, Tavares dos Santos C, Colonezi $\mathrm{GL}$, et al. Upper trapezius relaxation induced by TENS and interferential current in computer users with chronic nonspecific neck discomfort: an electromyographic analysis. J Back Musculoskelet Rehabil. 2015;28(1):1924; doi: 10.3233/BMR-140482.

23. Cheing GL, Hui-Chan CW. Analgesic effects of transcutaneous electrical nerve stimulation and interferential currents on heat pain in healthy subjects. J Rehabil Med.2003;35(1):15-19;doi:10.1080/16501970306101. 